\title{
Relação entre vitamina $D$ e cálcio no desenvolvimento do Diabetes Mellitus tipo 1 e 2 - Uma revisão de literatura
}

\author{
Paula Oliveira Dazini* \\ Carla Marcia Moreira Lanna* \\ Ana Paula Boroni Moreira
}

\section{RESUMO}

A prevalência de obesidade, associada a hábitos alimentares inadequados e sedentarismo tem aumentado a ocorrência Diabetes Mellitus, tornando-se um problema de saúde pública. Perspectivas imediatas na mudança do ambiente para reverter o aumento dos níveis de obesidade não são promissores, e existe uma necessidade de considerar outras opções para prevenir o diabetes. Tem sido apontada uma associação inversa entre os baixos níveis da vitamina D e cálcio com a incidencia do Diabetes Mellitus. Desse modo, a vitamina D e o cálcio têm sido propostos como um possível agente terapêutico para a prevenção e tratamento da doença. O objetivo do estudo foi realizar uma revisão de literatura sobre a influência da vitamina $\mathrm{D}$ e do cálcio na fisiopatologia do diabetes mellitus, assim como no controle glicêmico. Estudos têm mostrado que a vitamina $\mathrm{D}$ em conjunto com o cálcio são capazes de reduzirem o risco do desenvolvimento do diabetes mellitus tipo 2, enquanto que a insuficiência de vitamina $\mathrm{D}$ e de cálcio pode influenciar negativamente a glicemia. Além disso, nível plasmático de $25(\mathrm{OH}) \mathrm{D}$, tem sido correlacionado com o diagnóstico de Diabetes Mellitus tipo 1. Evidências recentes demonstram que a concentração sérica de vitamina $\mathrm{D}$ e do cálcio podem estar relacionados ao diabetes. Entretanto, estudos prospectivos e de intervenção em humanos que comprovem a efetividade de ambos, tanto na prevenção como no tratamento dessa doença, ainda são necessários.

Palavras-chave: Obesidade. Diabetes Mellitus. Vitamina D. Cálcio. Prevenção

\section{INTRODUÇÃO}

As prevalências de diabetes mellitus tipo 1 (DM1) e tipo 2 (DM2) estão aumentando cada vez mais, impulsionado principalmente pelo aumento dos níveis de obesidade (FRENCH; STORY; JEFFERY, 2001). Perspectivas imediatas para mudar o ambiente e reverter o aumento dos níveis de obesidade não são promissores, e existe uma necessidade de considerar outras opções para prevenir o diabetes.

A vitamina $\mathrm{D}$ tem sido associada com várias doenças, incluindo o DM 1 e DM 2. Tem-se observado que as pessoas com pré-diabetes e diabetes estabelecidas têm concentrações de $25[\mathrm{OH}] \mathrm{D}$ mais baixas no sangue que os pacientes com tolerância normal à glicose (MITRI; MARARU; PITTAS, 2011). Além disso, estudos de observação longitudinal têm associado níveis mais elevados de 25[OH]D com baixa prevalência de diabetes (AFZAL; BOJESEN; NORDESTGAARD, 2013).

Em relação ao cálcio, um estudo prospectivo mostrou uma associação inversa entre a baixa ingestão desse mineral com a incidencia de DM2 ou a síndrome metabólica (PEREIRA et al., 2002). Desse modo, a vitamina $\mathrm{D}$ e o cálcio têm sido propostos como um possível agente terapêutico para a prevenção e tratamento do diabetes mellitus.

Diante dos aspectos bioquímicos e metabólicos da vitamina $\mathrm{D}$ e do cálcio, o objetivo desta revisão foi trazer informações atualizadas sobre a influência desta vitamina ou hormônio e desse micronutriente na fisiopatologia do diabetes mellitus, assim como no controle glicêmico.

\section{REVISÃo DE LITERATURA}

Diabetes mellitus (DM) é um grupo heterogêneo de distúrbios metabólicos que apresenta em comum a hiperglicemia, resultante de defeitos na ação da insulina e/ ou na secreção de insulina (DIRETRIZES SBD, 2015).

* Universidade Federal de Juiz de Fora, Instituto de Ciências Biológicas, Departamento de Nutrição. E-mail: paula_dazini@hotmail.com

** Universidade Federal de Juiz de Fora, Instituto de Ciências Biológicas, Departamento de Fisiologia. 
A doença pode se manifestar através de sintomas como poliúria, polidipsia, perda de peso, polifagia, visão turva e por complicações agudas que podem levar a risco de vida, como a cetoacidose diabética e a síndrome hiperosmolar hiperglicêmica não cetótica. A hiperglicemia crônica está associada a dano, disfunção e falência de vários órgãos, especialmente olhos, rins, nervos, coração e vasos sanguíneos (GROSS et al., 2002).

No final da década de 1980, um estudo brasileiro estimou a prevalência do $\mathrm{DM}$, em $7,6 \%$ na população geral, variando de $2,7 \%$ entre indivíduos jovens até aproximadamente $17 \%$ naqueles com idade entre 60 e 69 anos (MALERBI; FRANCO, 1992). Dados mais recentes estimou que no Brasil $6,2 \%$ da população com 18 anos ou mais de idade referiram diagnóstico médico de diabetes, sendo $7,0 \%$ nas mulheres e de 5,4\% nos homens. Em relação à escolaridade, observou-se maior taxa de diagnóstico de diabetes $(9,6 \%)$ entre os indivíduos sem instrução ou com ensino fundamental incompleto (IBGE, 2013).

A classificação proposta pela Organização Mundial da Saúde (OMS) e pela Associação Americana de Diabetes (ADA) inclui quatro classes clínicas da doença, segundo a etiologia: Diabetes Mellitus tipo 1 (DM1), Diabetes Mellitus tipo 2 (DM2), outros tipos específicos do Diabetes Mellitus e Diabetes Mellitus gestacional (DIRETRIZES SBD, 2015).

O DM1 resulta da destruição das células betapancreáticas ocasionando a deficiência na produção de insulina. Em sua grande maioria, essa destruição de células beta é mediada por autoimunidade, contudo há casos em que não existem evidências de processo autoimune, sendo assim, referidos como forma idiopática da doença. O DM1 idiopático é caracterizado pela ausência de marcadores autoimunes contra as células betas (DIRETRIZES SBD, 2015).

Já o DM2 é uma doença metabólica que se caracteriza por defeitos na ação e/ou na secreção da insulina. Geralmente, ambos os defeitos estão presentes quando a hiperglicemia se manifesta, contudo, a doença inicia-se com resistência à insulina, defeito na ação da insulina e progride com o passar dos anos para a falência pancreática e deficiência na secreção de insulina (DIRETRIZES SBD, 2015).

Pertence à classificação dos outros tipos específicos do Diabetes Mellitus formas menos comuns de DM cujos defeitos ou processos causadores podem ser identificados. Já o Diabetes Mellitus Gestacional tratase de qualquer intolerância à glicose, de magnitude variável, com início ou diagnóstico durante a gestação (DIRETRIZES SBD, 2015).

O diagnóstico correto e precoce do pré-diabetes e do DM é extremamente importante porque permite que sejam adotadas medidas terapêuticas que podem evitar o desenvolvimento do diabetes nos indivíduos com pré-diabetes e retardar o aparecimento das complicações crônicas nos pacientes diabéticos (GROSS et al., 2002).

\subsection{Vitamina D e diabetes mellitus}

A vitamina $\mathrm{D}$ e seus pró-hormônios (colecalciferol, 25-hidroxicolecalciferol, 7-dehidrocolesterol, 24,25-dihidroxicolesterol, ergocalciferol e 1,25(OH) vitamina D), têm sido alvo de um número crescente de pesquisas nos últimos anos, demonstrando sua função além do metabolismo do cálcio e da formação óssea. Entre essas funções, tem-se a interação da vitamina $\mathrm{D}$ com outros sistemas como, por exemplo, o sistema imunológico, tendo em vista a expressão do receptor da vitamina D (VDR) e da enzima responsável pela formação de calcitriol (1-alfa-hidroxilase CYP27B1) em uma ampla variedade de tecidos corporais (JONES; TWOMEY, 2008).

Os seres humanos conseguem obter vitamina $\mathrm{D}$ através da exposição à luz solar, da sua dieta e através da suplementação (HOLLICK, 2007). Sendo a pele o único sítio capaz de produzir vitamina $\mathrm{D}$ (COZZOLINO; COMINETTTE, 2013). A produção endógena da vitamina se inicia na epiderme, onde está armazenada a sua substância precursora, o 7-dehydrocholesterol, que deriva do colesterol. A radiação solar ultravioleta $B$ (comprimento de onda, 290-315nm) penetra na pele e converte 7-dehydrocholesterol em pré-vitamina D3, que é rapidamente convertido em vitamina $\mathrm{D} 3$. A vitamina D2 é produzida através da irradiação ultravioleta do ergosterol de levedura, e a vitamina D3 através da irradiação ultravioleta de 7-desidrocolesterol de lanolina. Ambos são usados em suplementos de vitamina D (HOLICK, 2007).

A partir da dieta, a vitamina $\mathrm{D}$ pode ser adquirida através da ingestão de alimentos fontes como, fígado, leite, óleo de peixe, sardinha, atum, salmão e arenque. Ressaltando que em razão da sua lipossolubilidade, essa pode ter sua absorção reduzida quando a ingestão de lipídeos é insuficiente (COZZOLINO; COMINETTI, 2013).

A vitamina $\mathrm{D}$ obtida através da pele e da dieta é metabolizada no fígado em 25-hidroxivitamina D, sendo essa forma química utilizada para determinar concentrações séricas da vitamina de um indivíduo (status de vitamina D). Essa é preferível por manter níveis constantes e sua dosagem sérica ser bastante fidedigna. Além disso, sua meia-vida é de aproximadamente duas a três semanas, enquanto que a 1,25(OH)2D 3 possui meia-vida é de aproximadamente 6 horas e é fortemente influenciada por mecanismos 


\section{QuADRo 1:}

Síntese de 1,25 (OH) vitamina D

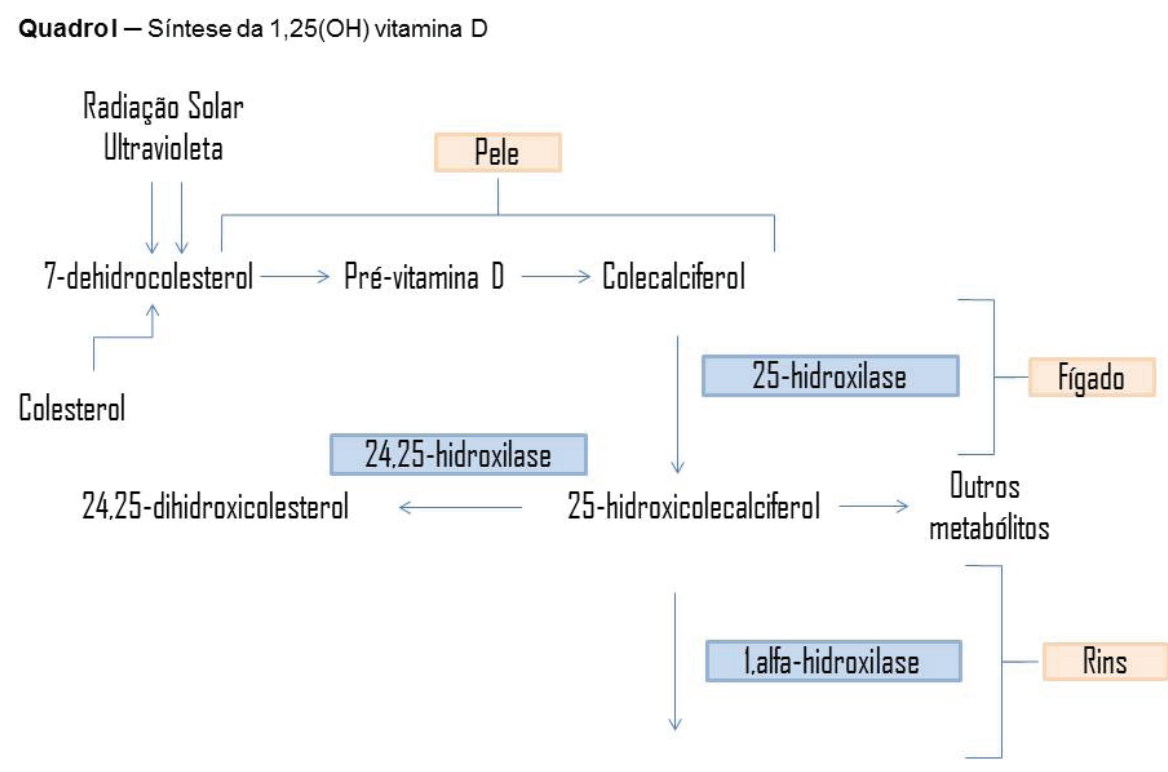

1,25 (매) vitamina D

Fonte: Adaptada PREMAOR (2006)

de retroalimentação (PREMAOR; FURLANETTO, 2006). Posteriormente a 25-hidroxivitamina D, ainda inativa, é metabolizada no rim pela enzima 25-hidroxivitamina D-1 $\alpha$-hidroxilase (CYP27B1) em sua forma ativa, a 1,25-di-hidroxivitamina $\mathrm{D}$ ou calcitriol (HOLICK, 2006).

O maior limitante da síntese de $25(\mathrm{OH}) \mathrm{D}$ parece ser a disponibilidade de vitamina D. Postula-se um mecanismo de retro-alimentação para $25(\mathrm{OH}) \mathrm{D}$, onde níveis baixos de cálcio estimulam a liberação do hormônio da paratireóide (PTH), que nos rins é responsável por estimular a hidroxilação da 25-hidroxivitamina $\mathrm{D}$ em sua forma ativa 1,25(OH)2D3, promovendo assim, aumento da reabsorção intestinal de cálcio. Além disso, a elevação de PTH estimula o aumento da reabsorção óssea de cálcio, regularizando sua concentração sanguínea (MOLINA, 2014).

Os níveis séricos da vitamina $\mathrm{D}$ tidos como adequado ainda são muito discutidos na literatura. Embora não haja um consenso sobre os níveis ideais da vitamina no soro, a deficiência de vitamina $\mathrm{D}$ é definida pela maioria dos especialistas como um nível da 25-hidroxivitamina D inferior a $20 \mathrm{ng} / \mathrm{ml}$ (50nmol/L) (HOLICK, 2006).

Hollis (2005) sugere que o nível ótimo de vitamina D seria aquele necessário para manter o PTH em níveis adequados, visto que a deficiência de vitamina $\mathrm{D}$ leva à diminuição do cálcio sérico, o qual, em consequência, estimula as glândulas paratireoides a liberar o PTH, a fim de elevar a reabsorção renal e óssea do cálcio.

Sendo assim, o nível de 25-hidroxivitamina D de 21 a $29 \mathrm{ng} / \mathrm{ml}(52$ a $72 \mathrm{nmol} / \mathrm{L})$ pode ser considerado um indicador de insuficiência relativa, e um nível de $30 \mathrm{ng} / \mathrm{ml}$ ou superior podem ser considerados como concentração suficiente da vitamina. A intoxicação é observada quando os níveis séricos de 25-hidroxivitamina D são maiores do que $150 \mathrm{ng} / \mathrm{ml}$ (374 nmol/L) (DAWSON-HUGHES et al., 2005).

Estima-se que 1 bilhão de pessoas no mundo têm deficiência ou insufuciência de vitamina $D$ (HOLLICK, 2006). Em nosso país, apesar da suposta maior exposição solar, baixas concentrações de $25(\mathrm{OH}) \mathrm{D}$ sérica também têm sido reportadas. Concentrações insuficientes da vitamina D foram observadas em $42 \%$ de idosos na cidade de São Paulo (SARAIVA et al., 2005). Em adolescentes saudáveis, a prevalência de insuficiência foi de $60 \%$, mesmo com $27,9 \%$ dos estudantes praticando atividade física ao ar livre, contudo apenas 14,9\% atendia a recomendação da ingestão da vitamina D (PETERS et al., 2009).

Os efeitos biológicos da forma ativa da vitamina D são mediados por seu receptor VDR, presente

HU Revista, Juiz de Fora, v. 43, n. 2, p. 163-172, abr./jun. 2017 
nos principais sítios de ação da vitamina. No núcleo das células-alvo, a 1,25(OH)2D3 se integra ao VDR. Esse complexo liga-se ao receptor de ácido retinóico (RXR), formando heterodímeros que atuam nos elementos-resposta da vitamina (VDRE), iniciando, então, uma cascata de interações moleculares que modulam a transcrição de genes específicos (KIMBALL; FULEIHAN; VIETH, 2008).

O marcante número de publicações que identificam uma inadequação na concentração sérica de vitamina $\mathrm{D}$ em todo o mundo tem despertado o interesse de pesquisadores, que frequentemente identificam a relação dessa vitamina não somente com a osteoporose, mas também com o desenvolvimento de doenças endócrino metabólicas (LIPPS, 2004).

A hipovitaminose $\mathrm{D}$ tem sido relacionada com mecanismos envolvidos na fisiopatologia de doenças crônicas, como DM 2, câncer e doenças cardiovasculares. A deficiência desta vitamina leva a diminuição da síntese e liberação de insulina, uma vez que uma das suas funções é atuar nas células B pancreáticas e na ação desse hormônio, sendo assim, sugerida sua participação na fisiopatologia do DM 2 (BELL, 2011).

Estudos atuais têm relacionado à deficiência de vitamina $\mathrm{D}$ com várias doenças autoimunes, incluindo DM 1, esclerose múltipla, doença inflamatória intestinal, lúpus eritematoso sistêmico e artrite reumatoide (LIPPS, 2004). Com isso associa-se a vitamina $\mathrm{D}$ como um fator extrínseco capaz de afetar a prevalência dessas doenças autoimunes.s.

\subsection{Cálcio e diabetes mellitus}

O cálcio é um elemento que desempenha um importante papel não só na mineralização óssea, mas também numa vasta gama de funções biológicas como, a contração muscular, mitose, coagulação sanguínea, transmissão do impulso nervoso ou sináptico e o suporte estrutural do esqueleto. Além disso, consumo adequado desse mineral tem sido apontado para prevenção de doenças como a osteoporose, hipertensão arterial, obesidade e câncer (HEANEY, 2006).

Nas últimas décadas estudos observacionais têm sugerido uma associação entre a homeostase anormal de cálcio com o risco de desenvolvimento de doenças crônicas, entre elas o DM 2, tendo em vista que a secreção da insulina e a resistência à sua ação tem mostrado depender dos níveis normais desse mineral (PITTAS; LAU; DAWSON-HUGHES, 2007).

Por não ser produzido endogenamente, a manutenção dos estoques corporais desse mineral ocorre por meio da ingestão de alimentos fontes como, produtos lácteos, vegetais de folhas verdes escuras, tofu, brócolis, sardinha, salmão, e através da suplementação. A necessidade de cálcio varia conforme a faixa etária, sendo maior em períodos de rápido crescimento como a adolescência $(1.100 \mathrm{mg} /$ dia), e na idade adulta, a necessidade diária de cálcio é em torno de $800 \mathrm{mg}$ (IOM, 2011).

A quantidade de cálcio absorvido é determinada pela ingestão e pela capacidade de absorção intestinal. Tem-se também a absorção saturável transcelular, dependente de vitamina $\mathrm{D}$, e a absorção não saturável intercelular, que é dependente da concentração de cálcio intraluminal (GRÜDTNER; WEINGRILL; FERNANDES, 1997).

Ao ser ingerido, o cálcio vai ser absorvido por transporte ativo (transcelular) dependente de $1,25(\mathrm{OH}) \mathrm{D}$ e do receptor de vitamina D (VDR), principalmente no duodeno onde há expressão significativa desses receptores, e em situações de baixa ingestão de cálcio. Quando a ingestão do nutriente é elevada, esse será absorvido por difusão passiva ao longo de todo o intestino delgado, por meio das tight junctions localizadas entre as células epiteliais (COZZOLINO; COMINETTTI, 2013).

Os mecanismos envolvidos na passagem do cálcio encontrado no lúmen para dentro das células devem-se aos canais apicais de cálcio, conhecidos como transient receptor potential cation channel (TRPV6 e TRPV5). Esses canais são regulados diretamente ou indiretamente pela vitamina $\mathrm{D}$ e pelo cálcio proveniente da alimentação, e são controlados por feedback pelo cálcio intracelular. Sendo assim, inicialmente o cálcio presente no lúmen entra nos enterócitos por meio dos canais de cálcio TRVP6 por difusão facilitada. Dentro da célula, o cálcio é sequestrado pela calbindina-D9k (proteína ligadora de cálcio induzida pela 1,25(OH)D). Ligado a essa proteína o cálcio é transportado pelo citoplasma em direção à membrana basolateral. Ao alcançar o outro lado da célula, ocorre o deslocamento em direção ao meio extracelular, mediado por Ca-ATPase de membrana plasmática ou, em menor escala, por $\mathrm{Na}$ / Ca (BRONNER, 2009).

O principal regulador da absorção transcelular de cálcio é a 1,25(OH)D, que age ao se ligar no seu receptor (VDR) na região promotora dos genes do TRPV5/6, da calbindina, no PMCa que é um cálcioATPase e no NCX1 que é um permutador sódio/ cálcio (COZZOLINO; COMINETTTI, 2013).

A manutenção das concentrações circulantes de cálcio é extremamente importante, e ocorre por meio de um sistema endócrino que envolve a 1,25(OH)D e seu receptor, o PTH e seu receptor, a calcitonina e o Car. As concentrações séricas totais desse mineral são reguladas para permanecerem entre 8,8 e 10,4mg/ 
$\mathrm{dL}(2,2$ e $2,6 \mathrm{mmol} / \mathrm{L})$. Se houver alterações nesses valores, a via hormonal de feedback negativo age para restaurar tais concentrações (PEACOCK, 2010).

As principais vias de excreção do cálcio são a urinária e a fecal. O conteúdo de cálcio não absorvido aparece nas fezes como cálcio fecal total. Já a excreção urinária está relacionada com o balanço entre a carga filtrada pelos rins e a eficiência da reabsorção pelos túbulos renais. Cerca de $98 \%$ do cálcio filtrado é reabsorvido ativamente ou passivamente (COZZOLINO; COMINETTE, 2013).

Segundo dados de consumo alimentar obtidos pela Pesquisa BRAZOS (Brazilian Osteoporosis Study) de 2007, 90\% dos entrevistados ingerem 1/3 $(400 \mathrm{mg})$ do valor preconizado de cálcio. E o uso de suplemento de cálcio foi referido por apenas $6 \%$ dos indivíduos (PINHEIRO et al., 2008).

Especula-se que a ingestão de cálcio inadequada pode alterar o equilíbrio entre os níveis de cálcio extracelulares e intracelulares, o que pode interferir na secreção normal de insulina, especialmente em resposta a uma carga de glicose, ou seja, as alterações no fluxo de cálcio podem ter efeitos adversos sobre a função de secreção das células beta pancreáticas (PITTAS; LAU; DAWSON-HUGHER, 2007).

O cálcio é essencial nos processos intracelulares mediados por insulina em tecidos responsivos a esse hormônio, tais como o músculo esquelético e tecido adiposo. As alterações no níves de $\mathrm{Ca}++$ em tecidosalvo primário da insulina podem contribuir para a resistência periférica à insulina através da dimuinuição da transdução de sinal de insulina que conduz a uma redução da atividade do transportador de glicose (GLUT-4) (OJUKA, 2004). Sendo assim, espera-se que a homeostase de cálcio anormal possa desempenhar um papel importante no desenvolvimento do DM2.

\section{DisCUSSÃo}

Na década de 1980 foi mostrado que a deficiência de vitamina $\mathrm{D}$ em roedores após a perfusão de glicose e arginina em um período de 30 minutos, obteve uma redução de $48 \%$ da liberação da insulina quando comparado ao grupo de roedores com valores reestabelecidos da vitamina (NORMAN et al., 1980). Mais tarde, a ligação entre a vitamina $\mathrm{D}$ e o diabetes foi reforçada pela descoberta VDR no pâncreas, mais especificamente nas células B produtoras de insulina, e também em vários tipos de células do sistema imune.

A vitamina $\mathrm{D}$ possui a capacidade de atuar nas células beta, assim como na proteína ligadora de cálcio dependente de vitamina $\mathrm{D}$ no tecido pancreático. Essa participa da resposta insulínica ao estimulo de glicose direta, através da ligação da 1,25(OH)2D3 ao seu receptor VDR na célula beta-pancreática, ou indiretamente, mediando o fluxo de cálcio nessas células (BARENGOLTS, 2010).

Pittas et al. (2006) conduziram um estudo prospectivo com mulheres não diabéticas, e verificaram que a vitamina $\mathrm{D}$ em conjunto com o cálcio são capazes de reduzirem o risco do desenvolvimento do DM 2, enquanto que a insuficiência de vitamina $\mathrm{D}$ e de cálcio pode influenciar negativamente a glicemia.

Para esclarecer a contribuição individual de cada nutriente com o DM 2, um grupo de pesquisadores examinou os efeitos combinados do total (comida e suplementos) de vitamina $\mathrm{D}$ e cálcio, e foi observado que, mulheres com a maior adequação de cálcio (1200 $\mathrm{mg} / \mathrm{d}$ ) e vitamina D (800 UI/d) apresentavam um risco $33 \%$ menor de desenvolver DM 2 em comparação com as mulheres com o menor adequação de cálcio $(600 \mathrm{mg} / \mathrm{d})$ e vitamina D (400 UI/d). Isso demonstra a importância de ambos os nutrientes como potenciais

\section{QUADRO 2:}

Adosorção intestinal de Cálcio

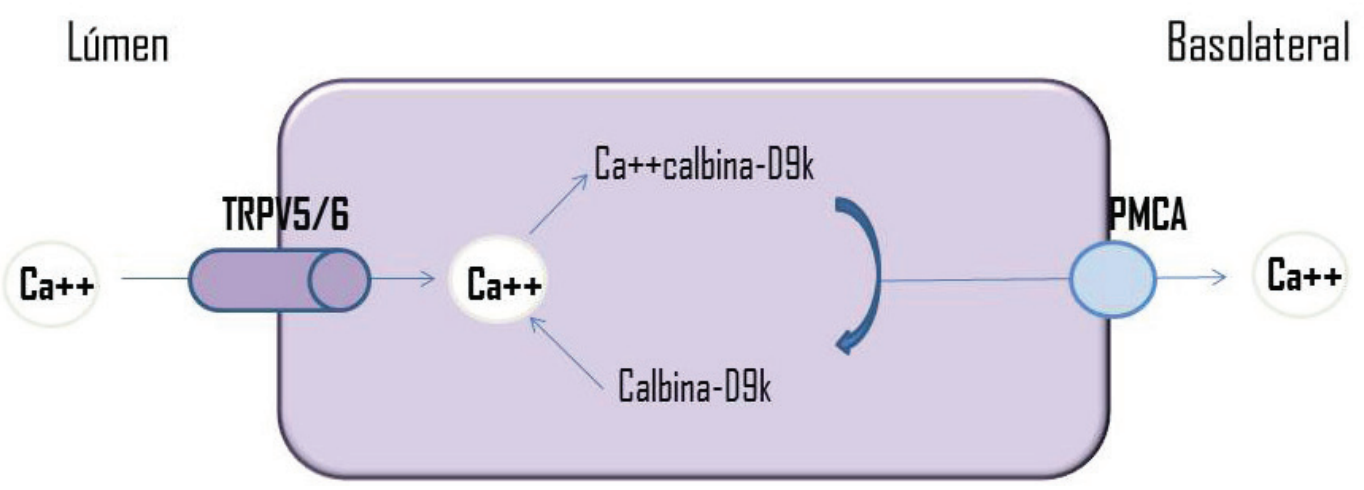

Fonte: Os autores (2016) 
modificadores de risco do tipo DM 2 (PIT'TAS, LAU, DAWSON-HUGHES, 2007).

Nikooyeh et al. (2011) apresentou um estudo onde a ingestão diária de uma bebida de iogurte enriquecido com vitamina $\mathrm{D}$ por 12 semanas, com ou sem adição de cálcio, melhorou as medidas antropométricas e estado glicêmico em indivíduos com DM 2.

O receptor VDR está presente em mais de 38 tecidos e participa do controle de genes vitais relacionados com danos oxidativos, doenças crônicas, inflamação e metabolismo ósseo. Sendo assim, a deficiência da vitamina $\mathrm{D}$ contribui para o aumento da resposta inflamatória associada à resistência a insulina em pacientes com DM 2. Um estudo recentemente mostrou que o aumento das citocinas pró-inflamatórias induz ao estresse nas células betapancreática, favorecendo apoptose dessas células (CHAGAS et al., 2012).

Em relação a função da vitamina $\mathrm{D}$ como nutriente anti-inflamatório, Gysemans et al (2005) apresentaram que a suplementação de vitamina D é capaz de aumentar a sensibilidade à insulina e diminuir processos de inflamação em ratos. Isso foi avaliado e verificado através da suplementação com calcitriol $(5 \mu \mathrm{g} / \mathrm{kg})$, sobre a síntese de citocinas inflamatórias e verificou-se a redução de citocinas IL-1beta e IL15. Foi identificado ainda o bloqueio da apoptose das células beta, o que contribuiu para melhora da sensibilidade à insulina.

Algumas pesquisas têm verificado a relação da ingestão de vitamina $\mathrm{D}$ e a sensibilidade à insulina de forma positiva (PITTAS et al., 2006). Em contrapartida, outros estudos não têm encontrado dados que associem a hipovitaminose $\mathrm{D}$ com o desenvolvimento do DM. Esse fato pode estar relacionado com o tamanho da amostra ou polimorfismo do receptor VDR, variáveis essas que contribuem de forma ativa para esses resultados (HIDAYAT; SEAT; SOEWONDO, 2010).

A suplementação com calcitrol em grupos populacionais que possuem concentrações normais dessa vitamina não possui efeito sobre o metabolismo de glicose. Entretanto, indivíduos que possuem estado de hipovitaminose $\mathrm{D}$, a suplementação sugere efeitos benéficos na síntese e secreção da insulina, o que parece contribuir para menor risco do desenvolvimento da doença (MITRI; MARARU; PITTAS, 2011).

Esses achados confirmam os resultados de um estudo de coorte finlandesa que apresentou uma associação inversa entre os valores basais de $25(\mathrm{OH})$ D e, após 17 anos, o risco de DM 2 (MATTILA et al., 2007). Contudo, vale ressaltar que a literatura ainda é muito escassa em relação à associação da deficiência da vitamina $\mathrm{D}$ com a presença do DM sendo necessários mais estudos sobre o tema.

Devido as propriedades imunomoduladoras da vitamina $\mathrm{D}$ e seus análogos, estudos tem sugerido que essas moléculas possam ser agentes potencialmente utilizados na prevenção, do DM1. A influência da vitamina no curso da doença tem sido atribuída também a sua interação com seu receptor VDR. Assim esse mecanismo agiria nas vias de sinalização responsáveis pela auto tolerância, culminando na redução da capacidade de apresentação de antígenos pelas células apresentadoras de antígenos (APC) (PENNA; ADORIN, 2000).

Um estudo evidenciou uma associação entre baixos níveis plasmáticos de vitamina $\mathrm{D}$ e o risco de desenvolvimento de DM1 (GREER et al., 2007). Littorin et al (2006) correlacionaram os níveis plasmáticos de $25(\mathrm{OH}) \mathrm{D}$, em adultos jovens, com o diagnóstico de DM1. Os resultados indicaram que a concentração de 25(OH)D era inferior nos indivíduos diabéticos, quando comparado ao grupo controle, sendo que $54 \%$ dos casos possuíam níveis de $25(\mathrm{OH})$ D inferiores a $32 \mathrm{ng} / \mathrm{mL}(80 \mathrm{nmol} / \mathrm{L})$.

Pozzilli et al. (2005) observaram que os níveis de $25(\mathrm{OH}) \mathrm{D}$ e $1,25(\mathrm{OH}) 2 \mathrm{D} 3 \mathrm{em}$ indivíduos diabéticos, no diagnóstico, eram consideravelmente inferiores aos níveis encontrados no grupo controle, independentemente do gênero, idade ou influência sazonal. Baumgartl et al. (1991) também encontraram tais resultados, porém neste caso tais diferenças verificaram-se sobretudo no período correspondente ao verão.

Pesquisas têm apontado que níveis mais baixos, tanto de $25(\mathrm{OH}) \mathrm{D}$ como de $1,25(\mathrm{OH}) 2 \mathrm{D} 3$, pode ser um fator importante no aumento da susceptibilidade para o desenvolvimento da DM1, possivelmente devido ao seu potente efeito modulador do sistema imunitário (Littorin et. al, 2006). Contudo, alguns estudos contradizem esta associação. Um estudo realizado na Florida, EUA, encontrou níveis reduzidos de $25(\mathrm{OH}) \mathrm{D}$, também durante o diagnóstico, porém essa não estava especificamente relacionado à DM1, uma vez que estes níveis se encontravam reduzidos tanto em indivíduos com DM1, recentemente diagnosticada, como no grupo controle ou indivíduos com DM1 já estabelecida (BIERSCHENK et al., 2009). No entanto, nenhum dos estudos acima mencionados efetuaram medições dos níveis de $25(\mathrm{OH}) \mathrm{D}$ ou $1,25(\mathrm{OH}) 2 \mathrm{D} 3$ antes do diagnóstico da doença.

Um estudo de coorte, realizado na Finlândia, sugeriu que o desenvolvimento de DM1 está associado à baixa ingestão de vitamina $\mathrm{D}$, durante $\mathrm{o}$ $1^{\circ}$ ano de vida, pois crianças que receberam $\geq 2000$ 
UI de forma regular, reduziram cerca de $80 \%$ o risco de desenvolvimento desta patologia (HYPPONEN et al., 2001). Em um outro estudo EURODIAB, houve uma redução no risco de desenvolver DM1 em 33\% nas crianças suplementadas (HEWISON et al., 2003).

Durante a gravidez, a suplementação com vitamina $\mathrm{D}$, através da dieta, também parece estar associada a uma diminuição do risco de aparecimento de auto anticorpos anti-insulina, anti-IA-2 e antiGAD em crianças com risco aumentado, tanto por genótipo HLA-DR, como por história familiar de DM1 (STENE; JONER, 2003). O aparecimento de autoimunidade é o primeiro sinal de destruição das células pancreáticas e decorre muito antes do diagnóstico clínico da doença.

Um estudo de caso-controle realizado por Stene et al. (2003) apresentou uma associação entre o consumo de óleo de fígado de bacalhau no primeiro ano de vida com um risco significativamente menor de DM1.

Uma meta-análise concluiu que a suplementação na infância parece ser protetora contra o desenvolvimento do DM1 (DANESCU; LEVY; LEVY, 2009). Já em adultos com a doença, uma redução na dose de insulina foi vista com a suplementação de calcitriol (JEFFERY et al., 2012).

Atualmente, mais de 2.000 análogos sintéticos da vitamina $\mathrm{D}$ são conhecidos, no entanto, o seu efeito hipercalcêmico restringe a sua utilização terapêutica (HUOTARI; HERZIG, 2008).

A carência de evidência do efeito benéfico da vitamina $\mathrm{D}$ deve-se muitas vezes a dosagem abaixo do ideal. Poucos estudos têm usado doses de vitamina D de, pelo menos, 2000 UI / dia. Outra razão para a falta de evidencias é o curto período de acompanhamento nas pesquisas. Para progressiva condições de melhora no estado pré-diabetes ou DM estabelecido, estudos de longo prazo são necessários para avaliar plenamente os benefícios da vitamina em uma intervenção, visando melhor elucidar os mecanismos de ação e as doses necessárias que possam apresentar os melhores benefícios (SEIDA et al., 2014).

O status de cálcio tem sido sugerido como mineral potencialmente importante no desenvolvimento do DM2. No estudo de Saúde da Mulher Negra, com uma coorte prospectiva com mais de 40 mil mulheres como idade entre 21-69 anos afro-americanas, sem ajuste da vitamina $\mathrm{D}$, observou-se uma relação contrária entre o consumo adequado de cálcio e o desenvolvimento do DM2 (VAN DAM et al., 2006). No estudo Nurses Health Study, essa relação entre a ingestão de cálcio total (alimentos e suplementos) também foi inversamente associado a doença (PITTAS et al., 2006).
A secreção de insulina em resposta a uma elevada concentração de glicose no plasma é um processo dependente de cálcio. As alterações nos níveis de secreção da insulina também foram envolvidas com distúrbios na homeostase da glicose no sangue e o aumento de cálcio citosólico tem sido associado com um aumento na expressão dos transportadores de glicose (GLUT4) em miócito, que, por sua vez, aumenta a atividade de transporte de glicose estimulada por insulina nestas células (PEREIRA et al., 2002).

A literatura tem apresentado a participação do cálcio na ação da insulina e tem mostrado que concentrações reduzidas deste mineral comprometem a ação periférica desse hormônio em função de alterações na atividade do transportador de glicose GLUT4. Sendo assim, concentrações elevadas de cálcio favorecem a ligação da calbidina ao receptor de insulina modulando a fosforilação da tirosina estimulada pela insulina (TAKIISHI et al., 2012).

Um estudo prospectivo Health Professionals Follow-up examinou a relação entre a ingestão de produtos lácteos e a incidência de DM 2 em 41.254 participantes do sexo masculino, sem história de diabetes durante 12 anos. Os autores concluiram que padrões alimentares caracterizados pela ingestão de produtos lácteos, especialmente a ingestão de laticínios de baixo teor de gordura, pode reduzir o risco de DM 2 em homens, e que cada aumento no consumo total de produtos lácteos que sirviam por dia estava associado com um risco mais baixo de $9 \%$ para o DM2 (CHOI et al., 2005).

Em contrapartida, uma pesquisa realizada com 863 indivíduos sem diabetes com idades de 40 a 69 anos pela Universidade do Texas, encontrou dados onde altas concentrações de cálcio sérico não necessariamente relacionadas com a ingestão de cálcio foram associadas com um risco aumentado do diabetes. Neste estudo foi medido a sensibilidade à insulina e resposta da insulina ao longo de um período de cinco anos, e encontraram a seguinte relação, indivíduos com a maior concentração de cálcio mostraram marcadores para um maior risco de diabetes (LORENZO et al., 2014).

Um outro estudodecoorte prospectivo demonstrou um aumento do risco de diabetes em indivíduos com aumento das concentrações de cálcio sérico. $\mathrm{O}$ objetivo do estudo foi investigar prospectivamente a associação entre as concentrações de cálcio sérico ajustado para a albumina e o risco de diabetes tipo $2 \mathrm{em}$ pacientes com alto risco cardiovascular. Após um período de acompanhamento médio de 4,78 anos, ocorreram 77 novos casos de diabetes tipo 2, e um aumento dos níveis séricos de cálcio durante o

HU Revista, Juiz de Fora, v. 43, n. 2, p. 163-172, abr./jun. 2017 
seguimento foi relacionado ao aumento do risco de diabetes (SALVADÓ et al., 2014).

Há evidências limitadas de um efeito da suplementação de cálcio nos parâmetros relacionados com o diabetes em estudos que examinaram os efeitos do cálcio isoladamente ou como um componente de produtos lácteos, contudo, mais estudos são necessários para estabelecer uma relação mais casual.

\section{Conclusão}

Evidências recentes demonstram que a concentração sérica de vitamina $\mathrm{D}$ e do cálcio podem estar relacionados ao diabetes. Entretanto, estudos prospectivos e de intervenção em humanos que comprovem a efetividade da adequação do status de ambos, tanto na prevenção como no tratamento dessa doença, ainda são escassos. Mais ainda, a compreensão dos mecanismos exatos pelos quais a $25(\mathrm{OH}) \mathrm{D}$ ou a forma ativa $1,25(\mathrm{OH}) 2 \mathrm{D} 3$ promovem melhor funcionamento das células- $\beta$ também são incompletos.

Sendo assim, os resultados de estudos futuros deverão definir o papel clínico de vitamina $\mathrm{D}$ e do cálcio como potenciais intervenções para prevenção ou tratamento do diabetes tipo 1 e tipo 2, que terão significativas implicações para a saúde, uma vez que tanto a vitamina $\mathrm{D}$ quanto o cálcio, podem ser implementados na prática clínica, através do consumo de alimentos fontes, exposição solar diária e, se necessário, suplementação dos mesmos.

\title{
Relationship between vitamin D and calcium in the development of Diabetes Mellitus type 1 and 2 - Review of the literature
}

\begin{abstract}
Currently, due to the increased prevalence of obesity, linked to inadequate eating habits and physical inactivity, diabetes mellitus has become a public health problem, increasingly common. Immediate prospects to change the environment to reverse the rising levels of obesity are not promising, and there is a need to consider other options to prevent diabetes. It has been suggested an inverse association between low levels of vitamin D and calcium with the incidence of diabetes mellitus or metabolic syndrome. Thus, vitamin D and calcium have been proposed as a possible therapeutic agent for the prevention and treatment of disease. The objective of the study was to conduct a literature review on the influence of vitamin D and calcium in diabetes pathophysiology, and glycemic control. Studies have shown that vitamin D in conjunction with calcium are able to reduce the risk of developing diabetes mellitus type 2, while the lack of vitamin $\mathrm{D}$ and calcium can adversely affect blood glucose. Furthermore, plasma levels of $25(\mathrm{OH}) \mathrm{D}$ has been correlated with a diagnosis of type 1 Diabetes Mellitus. Recent evidence shows that the serum concentration of vitamin D and calcium may be related to diabetes. However, prospective and intervention studies in humans to prove the effectiveness of both, both in prevention and in treatment of this disease are still needed.
\end{abstract}

Keywords: Obesity. Diabetes Mellitus. Vitamin D. Calcium. Prevention

\section{REFERÊNCIAS}

AFZAL, S; BOJESEN, S.E; NORDESTGAARD, B.G. Low 25-hydroxy vitamin $\mathrm{D}$ and risk of type 2 diabetes: a prospective cohort study and meta analysis. Clinical Chemistry, v. 59, n. 2, p.381-391, feb. 2013.

BARENGOLTS, E. Vitamin D and use for prediabetes. Endocrine Practice, v.16, n. 3, p. 476-485, may 2010.

BAUMGARTL, H.J. et al. Changes of vitamin D3 serum concentrations at the onset of immune-mediatedtype 1 (insulindependent) diabetes mellitus. Revista de Diabetes, v. 16, n. 3, p. 145-148, mar. 1991.

BELL, D. S. Protean manifestations of vitamin D deficiency, part 2: deficiency and its association with autoimmune disease, câncer, infection, asthma, derkmopathies, insulin resistance, and type 2 diabetes. South Medical Journal, v. 104, n.5, p. 335-339, may. 2011.

BIERSCHENK, L. et al. Vitamin D levels in subjects with and without type 1 diabetes residing in a solar rich environment. Diabetes Care, v. 32, n. 11, p. 1977-1979, nov. 2009.

BOSI, P.L. et al. Prevalência de diabetes mellitus e tolerância à glicose diminuída na população urbana de 30 a 79 anos da cidade de São Carlos, São Paulo. Arquivo Brasileiro de Endocrinologia e Metabologia, São Paulo, v. 53, n. 6, p. 726-732, ago. 2009.

BRONNER, E. Recent developments in intestinal calcium absorption. Nutrition Reviews, v. 67, n. 2, p. 109-113, feb. 2009. 
CHAGAS, C. E. et al. Focus on vitamin D, inflammation and type 2 diabetes. Nutrients, v. 4, n. 1, p. 52-67, jan. 2012.

CHOI, H.K. et al. Dairy consumption and risk of type 2 diabetes mellitus in men: a prospective study. Archives of Internal Medicine, v. 165, n. 9, p. 997-1003, may 2005.

COZZOLINO, S.M.F.; COMINETTI, C. Bases bioquímicas e fisiológicas da nutrição: nas diferentes fases da vida, na saúde e na doença. Vitamina D. In: COMINETTI, C. Cálcio. São Paulo: Manole, 2013. p. 178:89.

COZZOLINO, S.M.F.; COMINETTI, C. Bases bioquímicas e fisiológicas da nutrição: nas diferentes fases da vida, na saúde e na doença. Vitamina D. In: CHAGAS, C. E. A; MARTINI, L.A. Vitamina D. São Paulo: Manole, 2013. p. 413:416.

DANESCU, L.G.; LEVY, S.; LEVY, J. Vitamin D and diabetes mellitus. Endocrine, v. 35, n. 1, p. 11-17, feb. 2005.

DAWSON-HUGHES, B. et al. Estimates of optimal vitamin D status. Osteoporosis International, v. 16, n. 7, p. 713-716, jul. 2005.

DSBD. Diretrizes da Sociedade Brasileira de Diabetes: 20152016/Sociedade Brasileira de Diabetes ;- Rio de Janeiro: AC Farmacêutica, 2016. Disponível em $<\underline{\text { http: } / / \text { www.diabetes.org. }}$ br/sbdonline/images/docs/DIRETRIZES-SBD-2015-2016. pdf>. Acesso em 18 out 2016.

FRENCH, S.A.; STORY, M.; JEFFERY, R.W. Environmental influences on eating and physical activity. Annual Review of Public Health, v. 22, p.309 -335, may. 2001.

GREER, R.M. et al. Australian children and adolescents with type 1 diabetes have low vitamin D levels. Medical Journal of Australia, v. 187, n. 1, p. 59-60, jul. 2007.

GROSS, J.L. et al. Diabetes Mellitus: Diagnóstico, Classificação e Avaliação do Controle Glicêmico. Arquivo Brasileiro de Endocrinologia e Metabolismo, v. 46, n.1, fev. 2002.

GRÜDTNER, V.S.; WEINGRILL, P.; FERNANDES, A.L. Aspectos da absorção no metabolismo do cálcio e vitamina D. Revista Brasileira de Reumatologia, v. 37, n. 3, p. 143-151, jun. 1997.

GYSEMANS, C.A. et al. 1,25-Dihydroxyvitamin D3 modulates expression of chemokines and cytokines in pancreatic islets: implications for prevention of diabetes in nonobese diabetic mice. Endocrinology, v. 146, n. 4, p. 1956-1964, apr. 2005.

HEANEY, R.P. Calcium Intake and Disease Prevention. Arquivo Brasileiro de Endocrinololiga e Metabolismo, v. 50, n. 4, p. 685-693, ago. 2006.

HEWISON, M. et al. Differential regulation of vitamin D receptor and its ligand in human monocyte-derived dendritic cells. Journal Immunology, v. 170, n. 11, p. 5382-5390, jun. 2003.
HIDAYAT, R.; SEAT, S.; SOEWONDO, P. The association between vitamin $\mathrm{D}$ deficiency and type 2 diabetes mellitus in elderly patients. Acta Med Indonesiana, v. 42, n. 3, p. 123-129, jul. 2010.

HOLICK, M.F. Vitamin D Deficiency. The New England Journal of Medicine, v. 357, n. 3. p. 266-281, jul. 2007.

HOLICK, M.F. High prevalence of vitamin D inadequacy and implications for health. Mayo Clinic Proceedings, v. 81, n. 3, p. 353-373, mar. 2006.

HOLLIS, B.W. Circulating 25-hydroxyvitamin D level sindicative of vitamin D sufficiency: implications for establishing a new effective dietary intake recommendation for vitamin $\mathrm{D}$. The Journal of Nutrition, v. 135, n. 2, p. 317-322, feb. 2005.

HUOTARI, A,; HERZIG, K.H. Vitamin D and living in northern latitudes - an endemic risk area for vitamin $\mathrm{D}$ deficiency. International Journal of Circumpolar Health, v. 67, n. 2, p. 164-178, jun. 2008.

HYPPONEN, E. et al. Intake of vitamin D and risk of type 1 diabetes: a birth-cohort study. The Lancet, v. 358, p. 1500-1503, n. 9292 , nov. 2001.

IBGE. Pesquisa Nacional de Saúde 2013: percepção do estado de saúde, estilos de vida e doenças crônicas. Brasil, grandes regiões e unidades da federação. Rio de Janeiro, IBGE, p. 180, 2014.

(IOM) Institute of Medicine. Dietary Reference Intakes (DRIs) for calcium and vitamin D. Report at a glance 2011. Disponível em: <http://www.iom.edu/Reports/2010/ DietaryReferenceIntakesforCalciumandVitaminD/DRIValues. aspx $>$. Acesso em 26 de set de 2016.

ISAIA, G.; GIORGINO, R.; ADAMI, S. High prevalence of hypovitaminosis $\mathrm{D}$ in female type 2 diabetic population. Diabetes Care, v. 24, n. 8, p. 1496, aug. 2001.

JEFFERY, L.E. et al. Availability of 25-hydroxyvitamin D(3) to APCs controls the balance between regulatory and inflammatory T cell responses. Journal Immunology, v. 189, n. 11, p. 51555164, dec. 2012.

JONES, B.J.; TWOMEY, P.J. Issues with vitamin D in routine clinical practice. Rheumatology, v. 47, n. 9, p. 1267-1268, sep. 2008 .

KIMBALL, S.; FULEIHAN, G.; VIETH, R. Vitamin D: a growing perspective. Critical Reviews in Clinical Laboratory Sciences, v. 45, n. 4, p. 339-414, oct. 2008.

LIPPS, P. Which circulating level of 25-hydroxyvitamin D is appropriate? The Journal Steroid Biochemistry and Molecular Biology, v. 89, n. 1, p. 611-614, may. 2004.

LITTORIN, B. et al. Lower levels of plasma 25- hydrovyvitamin $\mathrm{D}$ among young adults at diagnosis of autoimmune type 1 diabetes compared with control subjects: Results from the nationwide

HU Revista, Juiz de Fora, v. 43, n. 2, p. 163-172, abr./jun. 2017 
Diabetes Incidence Study in Sweden (DISS). Diabetologia, v. 49, n. 12 , p. 2847-2852, dec. 2006.

LORENZO, C. et al. Calcium and phosphate concentrations and future development of type 2 diabetes: the Insulin Resistance Atherosclerosis Study. Diabetologia, v. 57, n. 7, p. 1366-1374, jul. 2014.

MALERBI, D.A.; FRANCO, L.J. Multicenter study of the prevalence of diabetes mellitus and impaired glucose tolerance in the urban Brazilian population aged 30-69 yr. The Brazilian Cooperative Group on the Study of Diabetes Prevalence. Diabetes Care, v. 15, n. 11, p. 1509-1516, nov. 1992.

MARQUES, C. D. L. et al. A importância dos níveis de vitamina D nas doenças autoimunes. Revista Brasileira de Reumatologia, v. 50, n. 1 , fev. 2010 .

MATTILA, C. et al. Serum 25-hydroxyvitamin D concentration and subsequente risk of type 2 diabetes. Diabetes Care, v. 30, n. 10, p. $2569-2570$, oct. 2007.

MITRI, J.; MURARU, M.D.; PITTAS, A.G. Vitamin D and type 2 diabetes: a systematic review. European Journal of Nutrition, v. 65, n. 9, p. 1005-1015, set. 2011.

MOLINA, E.P. Fisiologia Endócrina. 4. ed. Porto Alegre, RS: AMGH, 2014.

NIKOOYEH, B. et al. Daily consumption of vitamin D- or vitamin D + calcium-fortified yogurt drink improved glycemic control in patients with type 2 diabetes: a randomized clinical trial1-3. The American Journal of Clinical Nutrition, v. 93, n. 4, p. 764-771, apr. 2011.

NORMAN, A.W. et al. Vitamin D deficiency inhibits pancreatic secretion of insulin. Science, v. 209, p. 823- 825, aug. 1980.

OJUKA, E.O. Role of calcium and AMP kinase in the regulation of mitochondrial biogenesis and GLUT4 levels in muscle. The Proceedings of the Nutrition Society, v. 63, n. 2, p. 275-278, may. 2004.

PEACOCK, M. Calcium metabolism is health and disease. Clinical Journal American Society Nephrology, v. 5, n. 1, p. 23-30, jan. 2010.

PENNA, G.; ADORIN, L. 1alpha,25dyhidroxyvitamin D3 inhibits differntiation, maturation, activation and survival of dendritic cells leading to empaired alloreactive $\mathrm{T}$ cell activation. The Journal of Immunology, v.164, n. 5, p. 2405-2411, mar. 2000.

PEREIRA, M.A. et al. Dairy consumption, obesity, and the insulin resistance syndrome in young adults: the CARDIA Study. Journal of the American Medical Association, v. 287, n. 16, p. 2081-2089, apr. 2002.
PETERS, B.S.E. et al. Prevalence of vitamin D insufficiency in Brazilian adolescents. Annals of Nutrition and Metabolism, v. 54, n. 1, p. 15-21, feb. 2009.

PINHEIRO, M.M. et al. Nutrient intakes related to osteoporotic fractures in men and women - The Brazilian Osteoporosis Study (BRAZOS). Nutrition Journal, v. 8, n. 6, jan. 2008.

PITTAS, A.G. et al. Vitamin D and calcium intake in relation to type 2 diabetes in women. Diabetes Care, v. 29, n. 3, p. 650-656, apr. 2006.

PITTAS, A.G.; LAU, J; HU.; DAWSON-HUGHES, B. The Role of Vitamin $\mathrm{D}$ and Calcium in type 2 diabetes. A systematic Review and Meta-Analysis. The Journal of Clinical Endocrinology and Metabolism, v. 92, n. 6, p. 2017-2029, jun. 2007.

PREMAOR, M.O.; FURLANETTO, T.W. Hipovitaminose D em adultos: entendendo melhor a apresentação de uma velha doença. Arquivo Brasileiro de Endocrinologia e Metabolismo, v. 50, n. 1, p. 25-37, fev. 2006.

SARAIVA, G.L. et al. Influence of ultraviolet radiation on the production of 25 hidroxyvitamin $\mathrm{D}$ in the elderly population in the city of São Paulo (23 o 34'S), Brazil. Osteoporosis International, v.16, n. 12, p. 1649-1654, dec. 2005.

SEIDA, J.C. et al. Effect of vitamin D3 supplementation on improving glucose homeostasis and preventing diabetes: a systematic review and meta-analysis. The Journal of Clinical Endocrinology and Metabolism, v. 99, n. 10, p. 3551-3560, nov. 2014.

STENE, L.C.; JONER, G. Norwegian Childhood Diabetes Study Group. Use of cod liver oil during the first year of life is associated with lower risk of childhood on set type 1 diabetes: a large, population-based, case-control study. American Journal Clinical Nutrition, v. 78, n. 6, p. 1128-1134, jan. 2003.

TAKIISHI, T. et al. Vitamin D and diabetes. Rheumatic Disease Clinics of North America, v. 38, n. 1, p. 179-206, feb. 2012.

VAN DAM, R.M. et al. Dietary calcium and magnesium, major food sources, and risk of type 2 diabetes in U.S. black women. Diabetes Care, v. 29, n. 10, p. 2238-2243, out. 2006.

VAN DEN OORD, E.J. et al. Modeling with measured genotypes: effects of vitamin D receptor gene, age and latente genetic and environmental factores on bone mineral density. Behavior Genetics, v. 34, n. 2, p. 197-206, 2004.

Enviado em 17/11/2016

Aprovado em 09/08/2017 